Joseney Santos

\title{
Resposta brasileira ao controle da tuberculose
}

\section{Brazilian response to tuberculosis control}

\section{RESUMO}

A tuberculose ainda é um problema de saúde pública no Brasil. Em 2003, o controle da tuberculose foi destacado como prioridade dentre as políticas públicas de saúde. O artigo relata a resposta brasileira a esse desafio, descrevendo os principais componentes estratégicos contidos no Plano Nacional de Controle para o período 2003 a 2006. Dentre os principais resultados estão: expansão da cobertura da estratégia do Tratamento Supervisionado, aumento do percentual de cura de casos, redução do abandono ao tratamento e da taxa de incidência.

DESCRITORES: Tuberculose, epidemiologia. Tuberculose, prevenção e controle. Tuberculose, terapia. Prioridades em saúde. Planos e programas de saúde. Brasil.

\begin{abstract}
Tuberculosis remains a public health problem in Brazil. In 2003, controlling tuberculosis was highlighted as a priority among health public policies. The article reports the Brazilian response to this challenge, describing the main strategies of the National Control Plan for 2003 to 2006. Among its main results are: expansion of strategic coverage of the supervised treatment, increase in the percentage of cure, decrease in the number of patients' treatment default, and of the incidence rate.
\end{abstract}

KEY WORDS: Tuberculosis, epidemiology. Tuberculosis, prevention \& control. Tuberculosis, therapy. Health priorities. Health programs and plans. Brazil.

Tuberculose. Ministério da Saúde. Brasília,

DF, Brasil.

Correspondência | Correspondence:

Joseney Santos

SCS Quadra 4 Bloco A

Ed. Principal $3^{\circ}$ Andar PNCT

70304-000 Brasília, DF, Brasil

E-mail: joseney.santos@gmail.com 


\section{INTRODUÇÃO}

Em 1996, a Coordenação Nacional de Pneumologia Sanitária (CNPS) lançou o Plano Emergencial para o Controle da Tuberculose, cujo principal objetivo era aumentar a cobertura do Programa de Controle da Tuberculose (PCT) em 230 municípios, com ênfase ao diagnóstico e tratamento da doença. Esses municípios prioritários (MP) assim foram considerados devido à elevada carga da enfermidade.

Dois anos mais tarde, a CNPS lançou o Plano Nacional de Tuberculose, ${ }^{1}$ com a finalidade de ampliar as ações em todo o território nacional, adotando como metas diagnosticar pelo menos $92 \%$ dos casos esperados e tratar com sucesso pelo menos $85 \%$ dos casos diagnosticados, até 2001 .

Em 1999, o Ministério da Saúde (MS) formalizou a recomendação da implantação da estratégia do Tratamento Supervisionado (Directly Observed Treatment Strategy - DOTS) denominado no Brasil como TS-DOTS. ${ }^{2}$ Todavia, em função da estruturação e de mudanças no setor de saúde ocorridas naquele período, houve dificuldades para sua implantação.

Firmada em março de $2000^{3}$ durante a Conferência Ministerial sobre Tuberculose e Desenvolvimento Social, a Declaração de Amsterdã teve como pauta principal a busca de soluções para o controle da tuberculose (TB) em países em desenvolvimento. Uma vez identificados os principais fatores associados à doença, foram definidas linhas de ação para seu controle, considerando a importância do envolvimento da sociedade, utilização de tecnologia eficaz e acessível para o diagnóstico da TB, e incorporação dos serviços de atenção básica no atendimento aos pacientes com $\mathrm{TB}^{4}$

A partir de 2003, a TB foi colocada na agenda de prioridades das políticas públicas do Brasil. O Ministério da Saúde pactuou o fortalecimento da estratégia TSDOTS com as demais esferas de gestão como principal instrumento para alcançar as metas internacionais de detecção de $70 \%$ dos casos bacilíferos estimados e de cura de, pelo menos, $85 \%$ dos casos tratados. ${ }^{2, *}$

O atual Plano de Ação do Programa Nacional de Controle da Tuberculose (PNCT), aprovado em 2004, está fundamentado na descentralização e horizontalização das ações de vigilância, prevenção e controle da TB. A base para a implantação da estratégia TS-DOTS utiliza a força de recursos humanos das unidades de atenção básica, Programa de Saúde da Família (PSF) e Programa de Agentes Comunitários de Saúde (PACS). O PNCT está integrado ao Sistema Único de Saúde (SUS) e desenvolve as seguintes ações nos compo- nentes estruturais básicos: vigilância epidemiológica; medidas de proteção; integração com a atenção básica; ações integradas de educação em saúde, comunicação e mobilização social; capacitação e treinamento articulados com pólos de educação permanente; sustentação político-social; e avaliação, acompanhamento e monitoramento. Estes componentes têm suas atribuições definidas para as esferas federal, estadual e municipal.

A união tem atribuições normativas, de provimento de recursos financeiros; assessoria técnica e capacitação de recursos humanos para estados; avaliação, monitoramento e difusão de informação do País; desenvolvimento tecnológico; e política de aquisição, distribuição e controle de medicamentos e imunobiológicos. Os estados da federação desempenham o papel de assessoria técnica e capacitação de recursos humanos para municípios; realizam avaliação e monitoramento das ações e a difusão de informação do estado. Os municípios planejam e executam as ações de vigilância, prevenção e controle da TB em seus territórios.

\section{AÇÕES EXECUTADAS NO PROGRAMA NACIONAL DE CONTROLE DA TUBERCULOSE ENTRE 2003 E 2006}

O PNCT é estruturado em componentes distintos destinados a articular as ações de vigilância, prevenção e controle da TB com estados e municípios. Para tanto, é necessário que haja sustentação político-social para mobilizar os setores políticos para priorizar e assegurar a luta anti-TB. O apoio financeiro e a articulação intersetorial garantirão a execução das ações e atividades de vigilância e controle da $\mathrm{TB}$, permitindo que seja exercido o controle social das ações, evidenciando a importância do papel participativo da sociedade civil no controle da TB.

A inserção do PNCT nas atividades rotineiras da atenção básica dependia da priorização das ações de controle da TB por parte dos gestores, em qualquer uma das três esferas de governo, mostrando a disposição em se alcançar as metas propostas. Nesse sentido, foi adotada uma estratégia de mobilização dos gestores em oficinas macrorregionais com objetivo de sensibilizar, dimensionar responsabilidades e informar sobre os meios e recursos financeiros dedicados a essa atividade no âmbito do SUS. Desde 2004, vêm sendo realizadas oficinas buscando a mobilização política permanente.

As principais ações do PNCT estão baseadas na vigilância epidemiológica, prevenção e controle de casos. A vigilância tem como objetivo detectar precocemente

* Ministério da Saúde. Diretrzes do Programa Nacional de Controle da Tuberculose. Brasília. Disponível em http://portal.saude.gov.br/portal/ saude/visualizar_texto.cfm?idtxt=21446 [Acesso 20 abr 2007]. 
os casos de TB. Isso não pode ser alcançado de forma passiva e é fundamental que se estimule a busca ativa dos sintomáticos respiratórios, seja na comunidade ou em unidades de saúde, incluindo ambulatórios, emergências e hospitais nos grandes centros urbanos e em grupos de maior risco, tais como indígenas, albergados, população de rua, sistema prisional e portadores do HIV/Aids.

Para que a busca ativa seja efetiva, o PNCT tem como meta garantir todos os insumos necessários para a realização da baciloscopia de escarro de todos os sintomáticos respiratórios e cultura de escarro de todos os casos suspeitos de TB com baciloscopia negativa. A atenção deve ser redobrada e a cultura de escarro é fundamental nos pacientes infectados pelo HIV e naqueles suspeitos de TB multirresistente (TBMR). O oferecimento do teste sorológico anti-HIV é obrigatório para todo paciente com diagnóstico confirmado de TB, assegurando-lhe o aconselhamento voluntário pré e pós-teste. Assim como a busca ativa é ação básica para a detecção de casos entre os sintomáticos respiratórios, também será para os contatos intra e extradomiciliares. Reforça-se que essas ações devem primar pelo acolhimento, atitude que deve perpassar toda a equipe.

O Sistema de Informação de Agravos de Notificação (Sinan) é o sistema nacional adotado para o registro e processamento de dados de notificação e acompanhamento da TB. O Sinan é o pilar mais importante para as ações de planejamento, monitoramento e avaliação no controle da doença. A qualidade do sistema de informação está diretamente ligada à qualidade do preenchimento dos dados. Portanto, várias ações foram implementadas com este objetivo, como capacitar os profissionais dos serviços de saúde em sistemas de informação, visando promover a autonomia das coordenações estaduais e municipais do PNCT na gestão da informação do programa; promover o Sinan como sistema padrão para o registro de informações de notificação e acompanhamento de casos de TB, realizando a digitação e transferência vertical dos dados nos prazos estabelecidos pelas normas e rotinas do sistema; registrar e analisar periodicamente os casos de TB por meio do Livro de Registro e Controle de Tratamento dos Casos de Tuberculose nos serviços de saúde; utilizar os instrumentos de Notificação e Acompanhamento de casos (ficha de notificação/investigação de tuberculose e boletim de acompanhamento); registrar e acompanhar com instrumento de controle as pessoas submetidas ao tratamento da infecção latente; registrar os sintomáticos respiratórios na rede de laboratórios pelo Sistema de Informação Laboratorial de Tuberculose (SILTB) e contatos examinados no Sinan; tornar compulsória a notificação às secretarias municipais de saúde, por parte dos laboratórios públicos, conveniados e privados, dos resultados positivos de baciloscopia e culturas para Bacilo de Kock (BK).
Em relação ao tratamento e acompanhamento dos pacientes, o PNCT tem como metas tratar corretamente $100 \%$ dos casos diagnosticados de TB e curar pelo menos $85 \%$ desses. As estratégias devem, preferencialmente, ser desenvolvidas por equipes multiprofissionais e objetivar a inclusão social do paciente, ressaltando a importância da organização do serviço sob a estratégia TS-DOTS. Para tanto foi necessário elaborar, validar, normatizar e atualizar material técnico instrucional e educacional, junto com os pólos de educação permanente (PEP), os profissionais de saúde, os serviços e os usuários; organizar e divulgar a rede assistencial, identificando as unidades de saúde de referência e o fluxo de atendimento dos pacientes; organizar e divulgar a rede laboratorial, identificando os laboratórios de referência e estabelecendo fluxo de exames; fornecer esquemas de tratamento padronizados para todos os casos de TB; implantar e manter o controle de qualidade dos fármacos anti-TB (equivalência farmacêutica e farmacovigilância); monitorar e divulgar o cumprimento das normas nacionais para o tratamento de casos da TB nos estados e municípios; realizar e divulgar avaliações epidemiológicas e operacionais periodicamente; acompanhar, avaliar e divulgar as informações da co-infecção TB/HIV; capacitar profissionais de saúde na estratégia TS-DOTS, considerando a participação dos PEP.

As ações desenvolvidas pelos laboratórios de diagnóstico, acompanhamento e realização de teste de sensibilidade às drogas usadas no tratamento da $\mathrm{TB}$ são de fundamental importância para o PNCT. Assim, algumas ações foram programadas para a adequação $\mathrm{e}$ melhoria do diagnóstico dos casos de TB. São elas: a descentralização, sob a Coordenação dos Laboratórios Centrais de Saúde Pública (Lacen), do diagnóstico laboratorial da TB para laboratórios públicos e privados conveniados das capitais e MP e nos serviços de saúde que tenham o PCT implantado e que não desenvolvam estas atividades; ampliação da execução da baciloscopia para rede de serviços de saúde dos MP; implantação da cultura de escarro para identificação do BK e teste de sensibilidade em todos os Lacen; implantação do Sistema de Informação Laboratorial para Tuberculose (SILTB) nos Lacen e unidades laboratoriais dos MP; implantação de um programa de garantia da qualidade para os exames bacteriológicos na rede laboratorial; possibilitar a expedição do resultado da baciloscopia de escarro em até quatro horas na urgência/emergência e até 24 horas na rede ambulatorial.

\section{RESULTADOS DAS AÇÕES DO PROGRAMA NACIONAL DE CONTROLE DA TUBERCULOSE}

Para aperfeiçoar as ações e alcançar as metas foram priorizados 315 municípios em todo o País, de acordo com critérios essencialmente epidemiológicos e demográficos. Esses municípios concentram cerca de $80 \%$ do número de casos de TB no Brasil. 
Quanto à sustentação político-social, o PNCT promoveu a mobilização e sensibilização política dos gestores estaduais e municipais, disseminando a importância de reforçar as ações de controle da TB e implantar a estratégia TS-DOTS nos MP.

Com a adoção da estratégia baseada na organização de oficinas de gestores, foram alcançados expressivos resultados no monitoramento da evolução das ações do PNCT nos estados e municípios da federação, garantindo a expansão do TS-DOTS. Realizou-se 34 reuniões regionais para a discussão, monitoramento e avaliação do Plano Nacional de TB, incluindo as estratégias para a expansão do TS-DOTS especificamente em cada município; participaram os coordenadores dos 27 estados e dos 315 MP.

Em fevereiro de 2006, foi assinado o compromisso do Pacto pela Vida pelos gestores das três esferas do SUS. Das seis prioridades pactuadas, uma delas consiste no fortalecimento da capacidade de resposta às doenças emergentes e endemias, incluindo a TB.

No período compreendido entre 2004 a 2007, o governo brasileiro alocou 120 milhões de reais para as ações do PNCT. Esses recursos representam um aporte significativo, quase o dobro das cifras empregadas na última década. Além do montante investido pela União, houve a participação no financiamento de ações por parte dos estados e municípios. Seguindo essa tendência, é possível que haja um investimento ainda maior nas ações planejadas pelo PNCT para o controle da TB.

$\mathrm{Na}$ linha das ações prioritárias foram realizados cursos para os estados, municípios prioritários e o Distrito Federal sobre a operação do Sinan-TB. Os estados e municípios com maiores problemas de informação foram priorizados visando à melhoria da qualidade do sistema de informações sobre TS-DOTS. Para melhorar o acesso ao sistema de informação, 600 computadores e demais equipamentos de informática foram entregues aos serviços de saúde nos Estados, MP e Distrito Federal. Como estratégia de reconhecimento e valorização do esforço conjunto para a melhoria do controle da TB no País, destinou-se mais de dois milhões de reais como recursos extra teto, premiando os 229 municípios prioritários que encaminharam informações de encerramento dos casos acima de $90 \%$ nas coortes de 2004 , e para os estados que atingiram $75 \%$ dessa meta em 2006, por meio de repasse pela modalidade fundo a fundo.

Foram ministrados 313 cursos de capacitação e atualização, beneficiando 18.065 profissionais de nível superior, 39.168 de nível médio e 988 de nível elementar, num total de 58.221 profissionais da rede SUS, realizados pelo Ministério da Saúde, secretarias estaduais, municipais e do Distrito Federal, no período de 2003 a 2006.
Quanto ao objetivo de reforçar o diagnóstico de TB, foram realizados 27 cursos de formação para gerentes dos Lacen das unidades federadas, de formação de multiplicadores em baciloscopia e no SILTB, com replicação em todos os estados, e treinamento para 800 técnicos de laboratório. Os Lacen também receberam novos equipamentos de laboratório e de informática.

Reconhecendo a importância do fortalecimento das ações nos estados e municípios, principalmente considerando o planejamento, monitoramento e avaliação, o PNCT contratou 30 profissionais (consultores), denominados Força Tarefa. A função desses consultores é auxiliar os estados e municípios na implantação ou reforço das ações de controle da TB. Foi efetuado repasse de recursos para o fortalecimento e expansão da cobertura do PNCT e de estratégia TS-DOTS nos 315 MP. A proporção de serviços de saúde que implementaram a estratégia TS-DOTS passou de 7\% em 2000 para $81 \%$ em 2006, conforme os dados apresentados na Figura 1.

Atualmente, com a expansão da cobertura dos serviços que utilizam a estratégia TS-DOTS, o Brasil passou a pertencer ao grupo de países que possuem entre $50 \%$ a $90 \%$ dos serviços com a estratégia já implantada.

Em 2005 o Brasil alcançou as metas intermediárias de $73,5 \%$ de detecção dos casos estimados. Em relação à cura, o país alcançou a taxa de $69,3 \%$, se forem incluídos todos os casos da coorte, e de $75,4 \%$ se considerados apenas os casos com a situação de desfecho conhecida. Esta diferença se deve ao atraso nas informações de encerramento de casos. Para os casos de TB pulmonar bacilífera, a cura foi de 71,3\% e 76,7\% para todos os casos da coorte e para aqueles com informação de encerramento, respectivamente. No período de 1999 a 2005, evidenciou-se a redução do coeficiente de incidência de casos de TB, tanto o referente a todas as formas de apresentação clínica de TB $(5,7 \%)$, quanto à

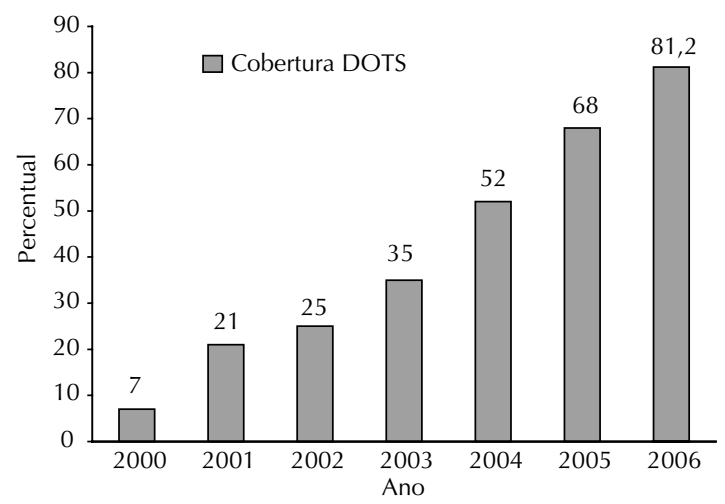

Fonte: secretarias estaduais de saúde

Figura 1. Proporção de serviços de saúde que adotaram a estratégia Tratamento Supervisionado - Directly Observed Treatment Strategy (DOTS). Brasil, 2000-2006. 


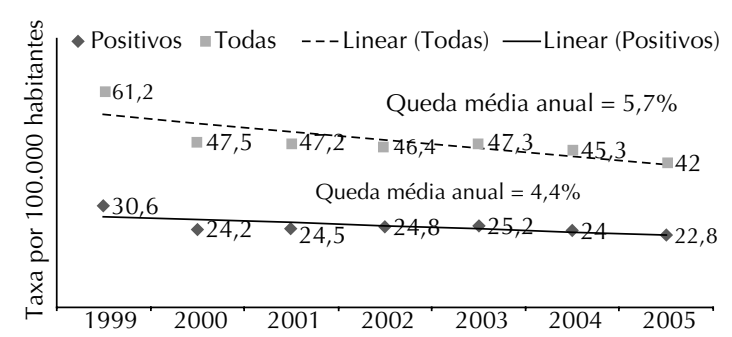

Fonte: Sistema de Informação de Agravos de Notificação/ Ministério da Saúde

Figura 2. Taxa de incidência de tuberculose referentes a todas as formas clínicas (Todas) de apresentação e a forma pulmonar bacilífera (Positivos). Brasil, 1999-2005.

forma pulmonar bacilífera $(4,4 \%)$, como pode ser observado na Figura 2. Caso esta tendência ora observada permaneça, a endemia de TB poderá ser conduzida ao controle adequado nesses locais.

A criação da Parceria Brasileira Contra a Tuberculose, em 2004, consubstanciou um marco importante da participação da sociedade civil organizada na luta contra a TB. Outras ações relevantes foram a interação com o Serviço Social da Indústria da Confederação Nacional das Indústrias (SESI/CNI) para o Controle da Tuberculose e a realização do Curso de Integração do PNCT com a Rede do Terceiro Setor e outros parceiros.

Quanto à divulgação em meios de comunicação pode-se apontar a campanha nacional de mídia: "A tuberculose tem cura", com informações à sociedade sobre a doença e o seu controle.

O acesso aos medicamentos vem sendo garantido com a aquisição e a distribuição nos quantitativos necessários (100 mil pacientes/ano, incluindo casos de TBMR). O processo foi avaliado por uma comissão da Organização Mundial de Saúde que atestou a evidência de um grande progresso nas ações desenvolvidas. Ao mesmo tempo foi implantado o Sistema de Controle de Qualidade dos Medicamentos de Tuberculose no Brasil.

Em 2004 o PNCT, com a assistência técnica da Organização Panamericana da Saúde (OPAS), iniciou o processo de elaboração da proposta ao Fundo Global de Combate à Aids, Tuberculose e Malária. Este fundo disponibilizou ao Brasil US\$27.240.000,00 para investimentos em 11 regiões metropolitanas de maior número de casos. Este projeto ora iniciado terá duração de cinco anos, tendo por objetivos a expansão do TS-DOTS, mobilização social, expansão do diagnóstico laboratorial com controle de qualidade e da co-infecção TB-HIV.

Entre as pesquisas operacionais está em curso o II Inquérito Nacional de Resistência aos Tuberculostáticos, que além de medir os níveis de resistência aos medicamentos utilizados no tratamento da doença em todas as capitais e municípios de grande porte, estimará a prevalência do HIV entre os portadores de TB.

\section{SITUAÇÃO ATUAL}

O Ministério da Saúde tem reafirmado a prioridade nas ações contra a TB, mobilizando os gestores do SUS ou repassando recursos adicionais aos estados e municípios. O MS tem colocado em prática um plano nacional que enfatiza a qualificação dos recursos humanos, a mobilização social, a qualidade da rede de laboratórios e o monitoramento e avaliação.

O maior desafio do PNCT está na expansão de cobertura de suas ações, tendo como base a estratégia TS-DOTS em todos os MP, além da diminuição do número de casos de abandono ao tratamento e o incremento da cura. Outros grandes desafios são o oferecimento e realização do teste anti-HIV para todas as pessoas maiores de 15 anos diagnosticados com TB; melhoria do sistema de informação, com vistas ao melhor planejamento das ações de vigilância e controle; busca pelo diagnóstico precoce de casos estimulando a captação e exames em sintomáticos examinados. $\mathrm{O}$ enfrentamento dessas prioridades possibilitará o fortalecimento das ações visando a redução do número de casos de TB na população geral e grupos vulneráveis.

O grande desafio para o SUS é aumentar sua abrangência de cobertura, tanto em relação ao número de unidades de serviço, como em relação ao número de pacientes sob TS-DOTS em todos os municípios.

Apesar da potencialidade existente, as 45.960 unidades ambulatoriais do SUS voltadas para a assistência básica ainda têm baixa cobertura do PNCT. Ressalte-se a importância da implantação das ações de controle da TB realizadas em conjunto entre o PNCT e a estratégia sanitária atenção básica com as equipes do PSF e o PACS, principalmente nas áreas onde a cobertura desta estratégia é mais expressiva, como no Nordeste e Centro-Oeste. Nas outras regiões do País, as unidades básicas de saúde tradicionais, com demanda espontânea, ainda têm grande importância estratégica e devem ser consideradas no contexto para definição das políticas do PNCT.

\section{CONCLUSÕES}

Avanços significativos puderam ser comprovados, como melhoria sensível do sistema de informações, maior avanço na expansão da estratégia TS-DOTS, melhoria nos indicadores de cura e diminuição gradativa de incidência e dos casos de abandono. A descentralização das ações de controle da TB, grande eixo estratégico do PNCT, avançou em velocidade menor, mostrando a necessidade da continuidade dos encaminhamentos políticos e institucionais para a sua plena efetivação. 
Apesar dos indicadores positivos, a endemia segue como grande problema da saúde pública pela sua capacidade de atingir com maior intensidade as populações marginalizadas. Além disso, é marcador social importante das precárias condições de sobrevivência das pessoas em quase todas as maiores cidades do País.

Esse cenário reflete a necessidade de se acoplar às estratégias já em execução, novas estratégias intersetoriais que visem à promoção da saúde e a melhoria da quali-

\section{REFERÊNCIAS}

1. Coordenação Nacional de Pneumologia Sanitária. Manual de Administração/Programa Nacional de Controle da Tuberculose. Bol Pneumol Sanit. 1996;4(1):7-56.

2. Ruffino-Netto A. Programa de controle da tuberculose no Brasil: situação atual e novas perspectivas. Inf Epidemiol SUS. 2001; 10(3): 129-38. dade de vida de segmentos da população, pilares fundamentais para o controle definitivo da TB no Brasil.

\section{AGRADECIMENTOS}

Ao Fábio Moherdaui e Dráurio Barreira, do Programa Nacional de Controle da Tuberculose - Ministério da Saúde, pelas observações técnicas; à Setsuko Santos, da Univesidade de Brasília, pelas sugestões.

3. World Health Organization. What is DOTS? A guide to understanding the WHO-recommended Tb control strategy known as DOTS. Geneva; 1999.

4. World Health Organization. Amsterdam Declaration to Stop Tb. In: The Ministerial Conference on Tuberculosis \& Sustainable Development; 2000 Mar 24; Amsterdam, The Netherlands; 2000. 